\title{
Removal of an Instrument Fractured by Ultrasound and the Instrument Removal System under Visual Magnification
}

\author{
${ }^{1}$ Alvaro Cruz, ${ }^{2}$ Claudia Gabriela Mercado-Soto, ${ }^{3}$ Israel Ceja, ${ }^{4}$ L Gerardo Gascón \\ ${ }^{5}$ Patricia Cholico, ${ }^{6}$ Claudia A Palafox-Sánchez
}

\begin{abstract}
Aim: The case of a lower molar with apical periodontitis, which had previous root canal treatment and a fractured instrument in the distal root beyond the foramen, is presented.
\end{abstract}

Background: The simultaneous presence of a foreign body (endodontic instrument or material) in periapical tissues and microorganisms in the root canal, are etiological factors in the formation or maintenance of a periapical lesion, and can lead to failure in endodontic treatment.

Case description: This instrument was removed through the staging platform technique, by using ultrasound and an Instrument removal system (IRS) microtube under microscope visual amplification. All the canals were re-instrumented, irrigated with sodium hypochlorite and passive ultrasonic irrigation, removal of smear layer and intracanal medication with calcium hydroxide for 8 days, after which they were filled. The symptoms disappeared and clinical and radiograph 2-year follow-up shows healing of periapical tissues.

Conclusion: The combined use of visual magnification microscope, ultrasound and the IRS system by staging platform technique, allowed the removal of an endodontic instrument beyond the foramen, which made it possible to apply a conventional disinfection protocol.

Clinical significance: Endodontic re-treatment by conservative approach of complicated cases it is an option with good clinical prognosis, before apical surgery or extraction.

Keywords: Fractured instruments, Instrument removal system, ultrasound, Endodontic retreatment, Apical periodontitis.

How to cite this article: Cruz A, Mercado-Soto CG, Ceja I, Gascón LG, Cholico P, Palafox-Sánchez CA. Removal of an Instrument Fractured, by Ultrasound and the Instrument

\footnotetext{
${ }^{1,6}$ Endodontic Postgraduate Program, Research Institute in Biomedical Sciences, CUCS, University of Guadalajara, México

${ }^{2,4}$ Endodontic Postgraduate Program, CUCS, University of Guadalajara, México

${ }^{3}$ Department of Physics, CUCEI, University of Guadalajara México

${ }^{5}$ Endodontics Postgraduate Program, CUALTOS, University of Guadalajara, México
}

Corresponding Author: Alvaro Cruz, Professor, Av. Francisco Javier Gamboa 230 Col. Arcos Sur, Guadalajara, Jalisco México, Zip code: 44150, Phone: +52-33 36159804, e-mail: endoacruz@yahoo.com
Removal System under Visual Magnification. J Contemp Dent Pract 2015;16(3):238-242.

\section{Source of support: $\mathrm{Ni}$ \\ Conflict of interest: None}

\section{INTRODUCTION}

The simultaneous presence of a foreign body (endodontic instrument or material) in periapical tissues and microorganisms in the root canal, are etiological factors in the formation or maintenance of a periapical lesion, ${ }^{1}$ and can lead to failure in endodontic treatment.

The main causes of instrument fractures is their overuse leading to metal fatigue, their incorrect kinematics, not knowing their physical properties, not discarding deformed files, and files with manufacturing defects, which act as force concentration zones. ${ }^{2}$

Faced with a fractured instrument inside the canal and a periapical lesion, the decision for a particular treatment depends on several factors, like the location of the fragment, the possibility of bypassing it or not and whether there is access to the apical portion of the canal. In the presence of a periapical lesion and a fractured instrument, the best is fragment removal by conservative approach. ${ }^{3,4}$ This allows standard protocols of root canal disinfection. If the fractured instrument impairs disinfection of the canal beyond the obstruction, the prognosis is affected. ${ }^{5}$ The inability to get access to disinfect the contaminated canal may lead to more invasive clinical maneuvers, such as periapical surgery. ${ }^{3}$

To remove a broken instrument, Ruddle ${ }^{6}$ proposed the 'staging platform technique', in which first a straight coronal and radicular access is created to visualize it, with modified Gates-Glidden bur. Using an ultrasound tip without irrigation at low power, dentin is removed progressively and carefully alongside the broken file, until exposing its coronal portion 2 to $3 \mathrm{~mm}$, or a third of the length thereof. With a thin ultrasound tip, the fractured instrument is touched, turning it counterclockwise, so that the ultrasonic vibration will release or unlock the file. If the fragment remains stuck, Ruddle ${ }^{6}$ recommends the use of a method of removal with microtube, as Instrument removal system (IRS) (Swiss Machining Inc, San Diego, USA). 
The case of a lower molar with apical periodontitis is reported, with a file fractured in the distal canal, beyond the foramen. The fractured instrument was removed with IRS system and ultrasound, under magnification with microscope. Re-treatment was performed with the protocol for these cases.

\section{CLINICAL CASE}

A 33-year-old female patient presents with discomfort on chewing on tooth 36 . She reports having received root canal treatment 5 years ago. The molar had provisional restoration. Vertical percussion tests were performed on all parts of the quadrant, referring mild pain in 36. Visual clinical inspection and palpation did not show inflammation signs in the adjacent gum or facial tissues.

In radiography root canal treatment was observed, with a radiopaque portion that resembled a fractured instrument (15 or 20 size) in the distal canal, beyond the apical foramen approximately $1.5 \mathrm{~mm}$, and periodontal ligament widened (Fig. 1A).

The presumptive diagnosis was previous endodontic treatment with signs of infection and chronic apical periodontitis, with secondary infection. The medical history did not contraindicate dental procedures. The treatment plan was removal of the fractured instrument and endodontic retreatment.

Mandibular block was given (mepivacaine $2 \%$ and epinephrine 1:100,000). The isolation with rubber dam was disinfected scrubbing with sodium hypochlorite $5.25 \%(\mathrm{NaOCl})$.

Temporary cement was removed with a \# 4 carbide bur at high speed. Gutta-percha was found in the pulp chamber, which was removed with a hot instrument until reaching the entrance of the canals. With Endo$\mathrm{Z}$ bur (Maillefer-Dentsply, Baillegues, Switzerland), coronary access was rectified and irrigated with $\mathrm{NaOCl}$. Under visual amplification (6x) with surgical microscope (OPMI1-FR, Carl Zeiss, Jena, Germany), two mesial canals and two distal canals were located. Distolingual canal was not previously instrumented, so it was explored with a \#15 K-file (Maillefer-Dentsply) to verify patency.

The gutta-percha from cervical and middle thirds of the mesial canals was removed with GPX \# 30 and \# 25 instruments (Brasseler, USA), in EndoMate engine (NSK, Japan) at 1,000 rpm. With \# 25 Hedström files (MailleferDentsply) gutta-percha of apical third was eliminated.

Cervical and middle thirds of the mesials and distolingual were flared with Gates-Glidden burs \# 3, \# 2, and \# 1 size. Files \# 20 were placed to establish the length in the three canals, by electronic method (Zx II Root Morita, Japan) and enlarged initially with Protaper Universal F1 and F2 (Maillefer-Dentsply) and apically until Flexofile \# 40 by balanced forces. $\mathrm{NaOCl} 3 \%$ was used as irrigant, with 30-gauge needle between each file. Sterile cotton pellet was placed at the entrance of the canals.

Gutta-percha from cervical and middle third of distobuccal canal was removed with GPX \# 30 and Hedström file \#25, until feeling a stop due to fractured instrument (Fig. 1B). Its most coronal portion was seen with the microscope. The guiding tip of Gates-Glidden drills 3 and 2 were cut-off using a low-speed diamond disk to prepare a flattened end at the maximum cross-sectional diameter of the Gates-Glidden burs. These modified Gates-Glidden were used into the canal at $600 \mathrm{rpm}$ and directed until it lightly contacted the most coronal part of the broken file. After creating the staging platform to get straight access, under $6 \times$ magnification, dentin alongside the fractured instrument was removed with ultrasound tip \# 4 (Pro-Ultra, Maillefer-Dentsply) dry at low power, until letting free $2 \mathrm{~mm}$ of fragment. Irrigation with $\mathrm{NaOCl}$ and drying of the operating field was made, to maintain visibility at all times.

A yellow IRS with an internal diameter of 0.4 and $0.6 \mathrm{~mm}$ outside was placed so that exposed portion of file fragment would remain within IRS side window. A radiograph was taken (Fig. 1C) to verify the proper position of the tip-fractured file within IRS. The internal shaft of IRS was withdrawn, by rotating it counter-clockwise (Fig. 1D). The IRS was pulled out and removed with the broken instrument, turning it counter-clockwise, to avoid screwing of the file. Canal length was established with apex locator and radiography. This canal was enlarged apically with Protaper Universal F4, and irrigation with $\mathrm{NaOCl} 3 \%$.

Passive ultrasonic irrigation (PUI) (Analytic, SybronEndo, Glendora, USA) was performed in the four canals, with a file 15 and $\mathrm{NaOCl}$, in three series of 20 seconds in each canal. The canals were flooded for 5 minutes with EDTA (REDTA, Roth Int, Chicago, USA), pumping it with a sterile \# 35 gutta-percha cone. Final irrigation was performed with $3 \mathrm{ml}$ of $\mathrm{NaOCl}$ and canals were dried with sterile paper cones (Pearson, California, USA). The canals were filled with calcium hydroxide (Sultan, Englewood, USA), mixed with double-distilled water, in thick paste consistency by lentulo spiral size 35 to $20.000 \mathrm{rpm}$. The excess paste was removed and the coronal access sealed with Cavit R (3M Espe, Seefeld, Germany). Ibuprofen $400 \mathrm{mg}$ was prescribed if postoperative discomfort presented. The next appointment was scheduled at 8 days. The IRS microtube with the fractured portion of file was revised on scanning electron microscope (Figs 2A and B). 

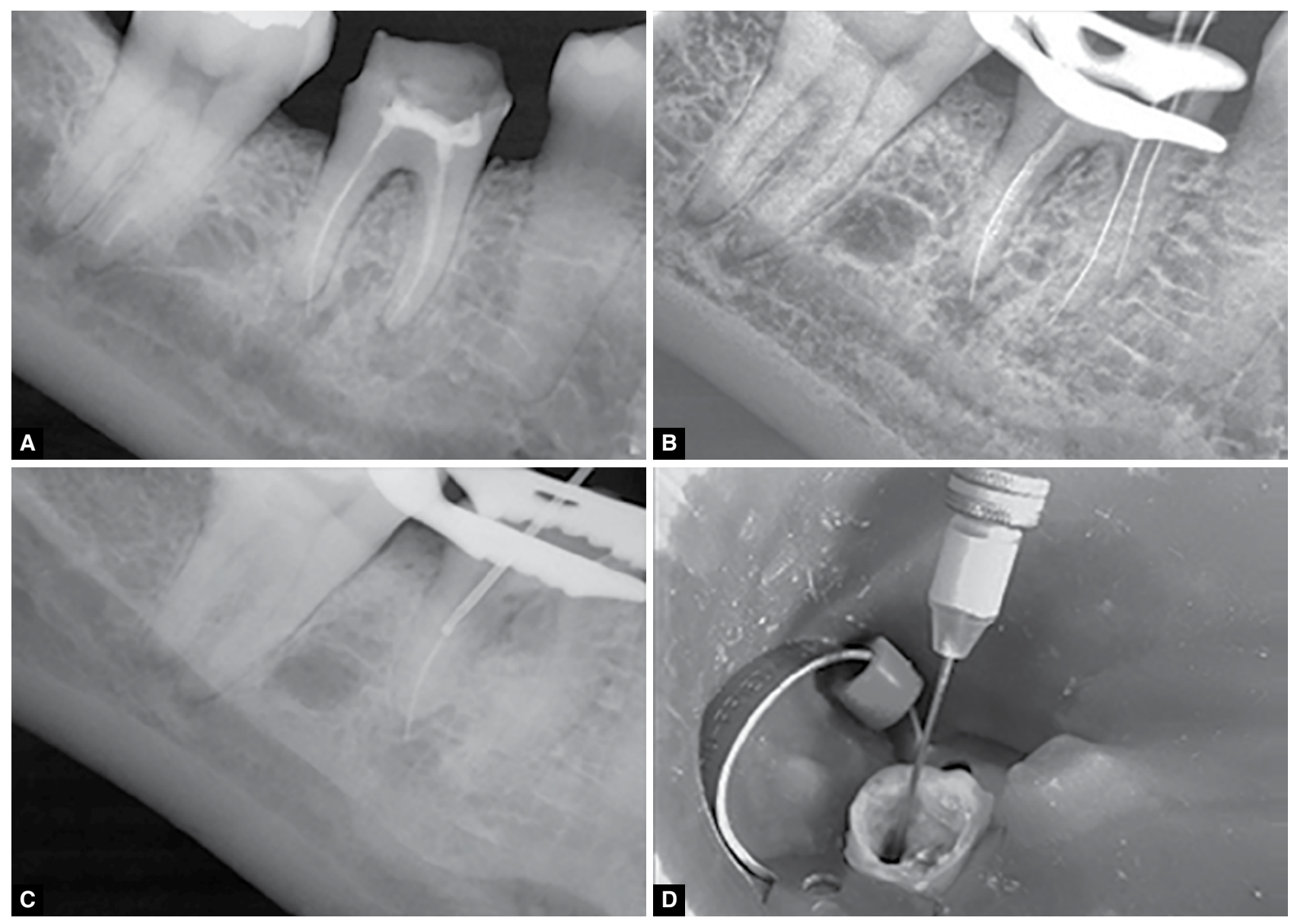

Figs 1A to D: (A) The radiograph shows the instrument in the distal fractured root beyond the foramen, (B) Desobturation X-ray, (C) Fractured instrument is observed within the IRS tube and (D) Clinical image of IRS instrument in position

At the next appointment, the patient reported no symptoms, was anesthetized and isolated as described and coronary seal removed with high-speed bur \# 4 . Calcium hydroxide paste was stirred up with irrigation of $20 \mathrm{ml}$ of $\mathrm{NaOCl}$ and filing with the master files. Ultrasonic irrigation again was performed as described, the canals flooded with EDTA for 5 minutes and dried with sterile paper points. They were filled with gutta-percha points sterilized and AH Plus (Dentsply-Maillefer) using hybrid Tagger condensation technique with a Gutta-condensor size 45 (Maillefer-Dentsply) at 20,000 rpm. Guttapercha excess was removed, vertical condensation was

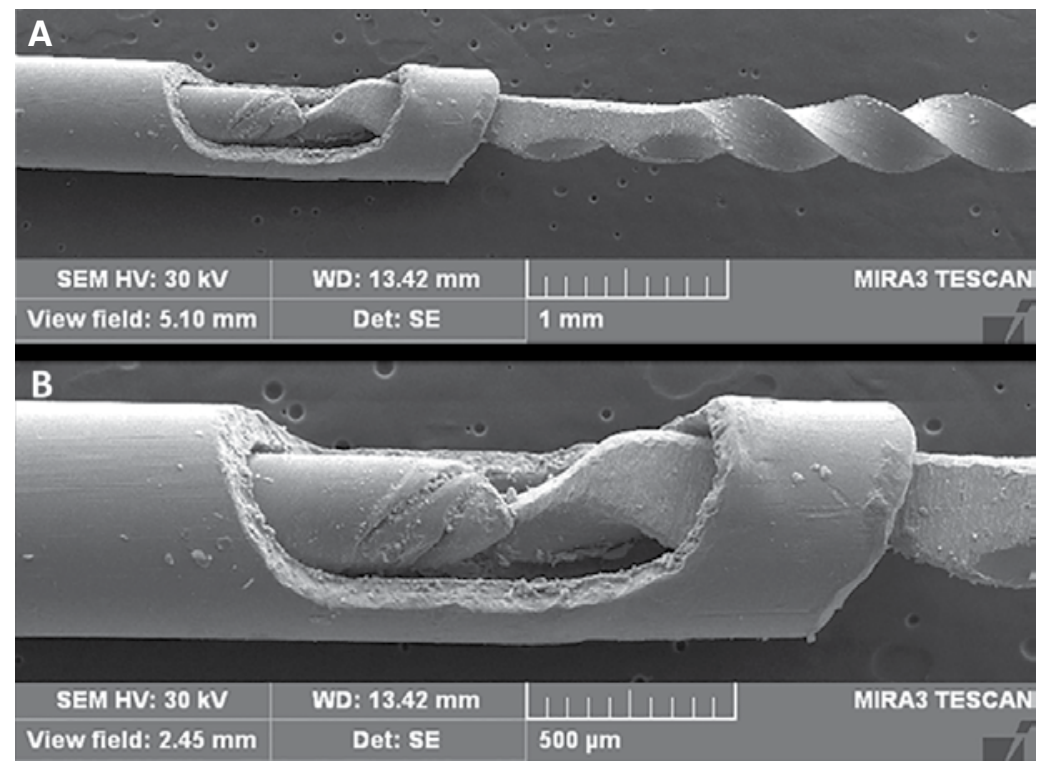

Figs 2A and B: Scanning electron microscope image with the file attached to IRS, $37 \times$ and $139 \times$. The internal shaft presses the tip of the fractured file; part of its spirals is carved by ultrasound 

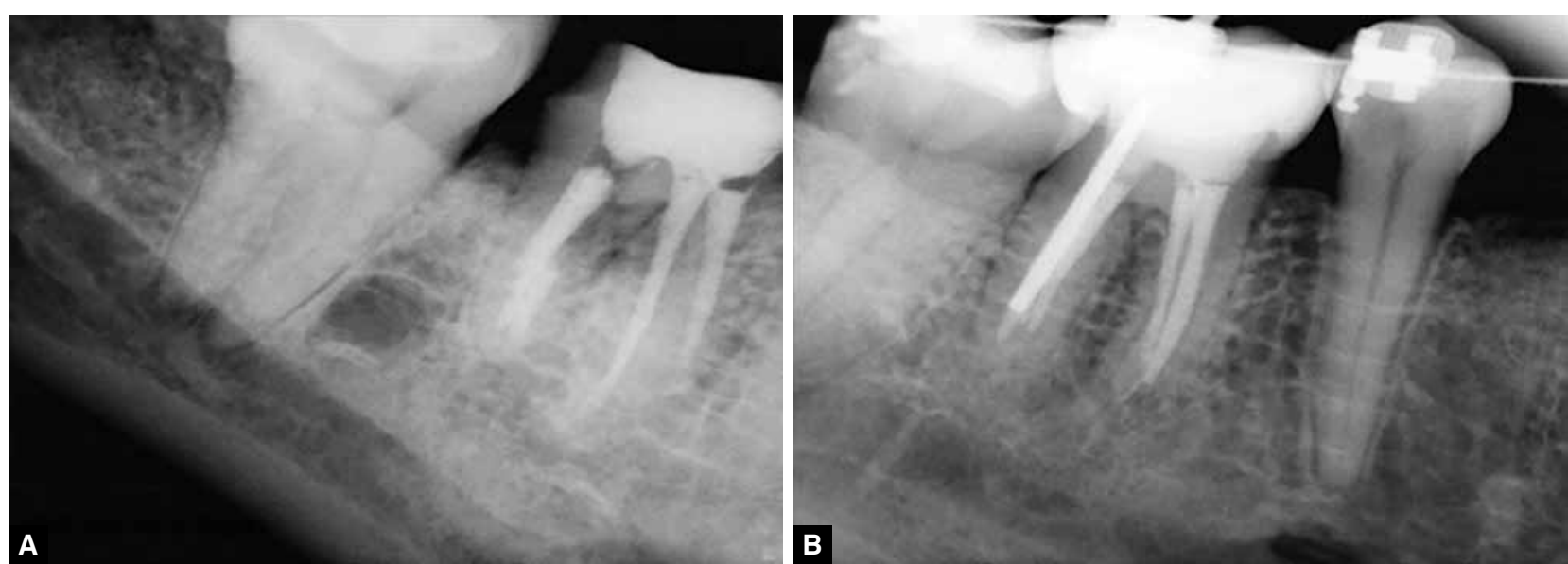

Figs 3A and B: (A) Final X-ray, where the DB canal was left partially filled to post. (B) X-ray control at 2 years. Molar is restored and periapical structures look normal

performed and the pulp chambers were cleaned of excess with sterile alcohol swab. Cavit $\mathrm{R}$ was placed to seal the coronary access. Gutta-percha in cervical and middle third was retired from disto-buccal canal (Fig. 3A) for post. Immediate restoration was indicated.

In clinical and radiographic control at 2 years, patient was asymptomatic and previous small lesion in distal root is healed. Patient was under orthodontic treatment (Fig. 3B).

\section{DISCUSSION}

Lighting and magnification with a microscope are helpful and indispensable for the treatment of these cases. The microscope-ultrasound approach can significantly increase the chances of success in the procedures, ${ }^{5-8}$ as the tips of ultrasound can be used safely in deep areas of the canal, alongside the fractured instrument. Without the microscope or staging platform, it is easy to contact the top of the fragment and to push it further into the canal. ${ }^{10}$ The fact that the fractured portion of the instrument is more than $5 \mathrm{~mm}$ improves the prognosis for remotion, ${ }^{9}$ since the staging platform can be done and having a greater length to catch it with the IRS, as in this case.

The entrances of others canals must be blocked, because ultrasound can remove the fragment of the instrument, and it may fall and block another canal. ${ }^{10}$ Also, the clinician should have prior training in the use of thin ultrasonic tips deep in the canals, and follow these procedures calmly and without being stressed, ${ }^{9}$ due to the concentration that this procedure requires. These thin ultrasonic tips must be used dry to ensure a direct vision, and on low power to avoid the fracture of the ultrasonic tip. ${ }^{10}$

Microtube systems are an option to retreat a fractured instrument when ultrasound cannot remove it. ${ }^{8}$ The diameter of the microtube to be used is decided by its external caliber, since it indicates how deep it can be safely introduced. ${ }^{6}$ However, the IRS has several limitations, such as the risk of perforation, ledges or root weakening, so the clinician must evaluate their use in thin walls or roots with large concavities, ${ }^{3}$ since the excessive wear can influence the outcome of treatment. ${ }^{5}$ In the posterior region, it may be difficult to use them due to a lack of space. ${ }^{6}$ Furthermore, removal of fractured instruments located beyond the curvature has limited success rate, ${ }^{9-11}$ and can lead to excessive wear and a reduced resistance of remaining dentin and root perforations, ${ }^{4}$ it should, therefore, be assessed for its risk-benefit.

In this case, the removal of the broken instrument allowed accessing the apical portion, and establishing a clinical protocol for cases with apical periodontitis, ${ }^{12,13}$ which includes enlarging the canal, irrigation with $\mathrm{NaOCl}$ and PUI, smear layer removal and placement of $\mathrm{Ca}(\mathrm{OH})_{2}$ as intracanal medication. Also, a canal that had not been treated was located, which by itself created or maintained the periapical lesion. ${ }^{1}$

\section{CONCLUSION}

The combined use of visual magnification microscope, ultrasound and the IRS system by staging platform technique, allowed the removal of an endodontic instrument beyond the foramen, which made it possible to apply a conventional disinfection protocol. These tools with training are valuable aids in the resolution of complex cases, and avoid more invasive surgical procedures. Proper disinfection and sealing of contaminated canals are fundamental for the establishment of better conditions of periapical healing. ${ }^{12}$

\section{REFERENCES}

1. Nair PNR. Pathogenesis of apical periodontitis and the causes of endodontic failures. Crit Rev Oral Biol Med 2004;15:348-381.

2. Lópes H, Siqueira JF Jr, Elias CN. Mecanismos de fratura dos instrumentos endodônticos, chapter in Endodontia, biología e técnica: Lópes H, Siqueira JF Jr, editors. Guanabara Koogan, SA Rio de Janeiro Brasil 2004. p. 483-494. 
3. Zuolo M, Kherlakian D, Mello J, Cohelo M, Cabral M. Re-intervención en Endodoncia. Ed Santos, São Paulo Brazil 2012. p. 44-45.

4. Nagai O, Tani Y, Kayaba Y, Kodama S, Osada T. Ultrasonics removal of broken instruments in root canals. Int Endod J 1986;19:298-304.

5. Souter N, Messer H. Complications associated with fractured file removal using an ultrasonic technique. J Endod 2005;31: 450-452.

6. Ruddle C. Nonsurgical retreatment. J Endod 2004;30:827-845.

7. Plotino G, Pameijer C, Grande N, Somma F. Ultrasonics in endodontics: a review of the literature. J Endod 2007;33:81-95.

8. Suter B, Lussi A, Sequeira P. Probability of removing fractured instruments from root canals. Int Endod J 2005;38:112-123.

9. Hülsmann M, Schinkel I. Influence of several factors on the success or failure of removal of fractured instruments from the root canal. Endod Dent Traumatol 1999;15:252-258.
10. Ward JR, Parashos P, Messer HH. Evaluation of an ultrasonic technique to remove fractured rotary nickel-titanium endodontic instruments from root canals: an experimental study. J Endod 2003;29:756-763.

11. Ward JR, Parashos P, Messer HH. Evaluation of an ultrasonic technique to remove fractured rotary nickel-titanium endodontic instruments from root canals: clinical cases. J Endod 2003;29:764-767.

12. Ricucci D, Russo J, Rutberg M, Burleson JA, Spångberg LS. A prospective cohort study of endodontic treatments of 1,369 root canals: results after 5 years. Oral Surg Oral Med Oral Pathol Oral Radiol and Endod 2011;112:825-842.

13. Estrela C, Alencar AHG, Decurcio DA, Borges AH, Guedes OA, Estrela CRA. Influência de estratégias de sanificação no sucesso do tratamento da periodontite apical. Rev Odontol Bras Central 2012;21:367-375. 